\title{
Eddy Current Loss Model for Ferrite Ring Cores based on a Meta-Material Model of the Core Properties
}

\section{Journal Article}

Author(s):

Dimier, Théophane (1D; Biela, Jürgen (1)

Publication date:

2022-02

Permanent link:

https://doi.org/10.3929/ethz-b-000487530

Rights / license:

In Copyright - Non-Commercial Use Permitted

Originally published in:

IEEE Transactions on Magnetics 58(2), https://doi.org/10.1109/tmag.2021.3084812 


\title{
Eddy Current Loss Model for Ferrite Ring Cores based on a Meta-Material Model of the Core Properties
}

\author{
Théophane Dimier, Student Member, IEEE, and Jürgen Biela, Senior Member, IEEE \\ Laboratory for High Power Electronic Systems, ETH Zürich, Switzerland
}

\begin{abstract}
A model for estimating the total eddy current losses in ring ferrite cores is proposed, which is based on a microstructure model of the electrical parameters of the material (conductivity and permittivity) and on the solution of the wave equations in the core. The presented model, combined with state of the art estimation of the hysteresis losses gives good agreement with the core loss measurements, over a wide range of frequency $(10 \mathrm{kHz}$ to $700 \mathrm{kHz})$.
\end{abstract}

\section{Index Terms-Core Losses, Eddy Currents, Ferrite, Power Electronics}

\section{INTRODUCTION}

Ferrite cores are widely used in power electronics [1], among other applications, to build magnetic components as inductors or transformers. They offer low losses at high frequencies and a decent saturation flux density (around $0.4 \mathrm{~T}$ for MnZn ferrites) at reasonable costs. With wide-band gap semiconductors, efficient converters with increasing switching frequencies beyond $100 \mathrm{kHz}$ are enabled [2], which could lead to potential higher core losses in magnetic components. For minimizing the losses and optimally designing the converter and its magnetics, accurate models for the core losses are required [3].

The core loss models can be categorized as empirical based or physics based models. The first category contains also the well-known Steinmetz Equation (SE). It was originally derived for electrical steel cores and is composed of two terms [4]:

$$
P=k_{h} f B^{1.6}+k_{E C} f^{2} B^{2}
$$

with $P$ being the core loss power density, $k_{h}$ the hysteresis loss coefficient, $B$ the flux density, $k_{E C}$ the eddy current loss coefficient, and $f$ the frequency. The Steinmetz equation is often modified to a compact single term expression [5]:

$$
P=k f^{\alpha} B^{\beta}
$$

To consider some excess losses that are not included in the two terms of the complete SE, a third term has been proposed by Bertotti [6].

In all these models, sinusoidal waveforms are assumed. Since in most converters the excitation is not sinusoidal, extensions have been proposed to adapt the SE to rectangular excitations by adding a coefficient depending on the duty cycle of the excitation (Waveform Coefficient SE or Rectangular Excitation SE) [7]. Furthermore, generalisations of the SE have been developed to allow the use of its parameters with any waveform (GSE, iGSE, $i^{2} \mathrm{GSE}$ ) [5].

Core losses can also be estimated based on physical models, which typically rely on modelling the low frequency hysteresis with the Preisach model [8] or the Jiles-Aetherton model [9]. An extension of the latter has been derived by its original authors to include the effect of conduction and eddy current on the overall hysteresis [9].

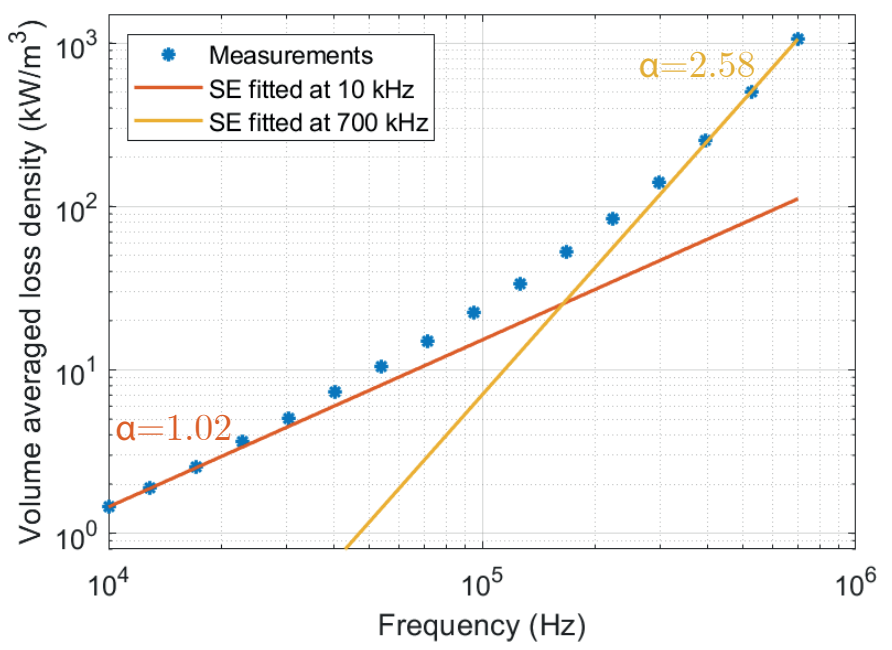

Fig. 1. Steinmetz equation fails at capturing the frequency scaling of losses as shown by the variation of the parameter $\alpha$. Frequency dependence becomes over-quadratic at high frequency. Measurement points were obtained as described in section IV with a peak to peak flux density of $100 \mathrm{mT}$.

State of the art methods have nevertheless some drawbacks. First, they all require a knowledge of the flux density everywhere in the core. This is usually not a problem when the frequency remains relatively low, so that the flux distribution is similar as at DC.

However, the permittivity of ferrites is not negligible and its impact on flux distribution becomes prominent when the frequency increases as standing waves can occur [10]. The flux distribution is no longer similar as at DC. Second, with increasing frequency, the standing waves typically result in variation of the effective permeance of the core as well as in potential macroscopic eddy currents [11]. Those induced currents are then causing additional losses meaning that the frequency scaling of the total core losses changes. This phenomenon is described in Fig. 1, where the frequency scaling coefficient $\alpha$ of the compact SE is around 1 at low frequency (indicating a linear influence of the frequency) and more than 2.5 at $700 \mathrm{kHz}$.

The occurrence of standing waves at high frequencies depends on the electro-magnetic parameters of the material, but conductivity and permittivity are typically frequency de- 


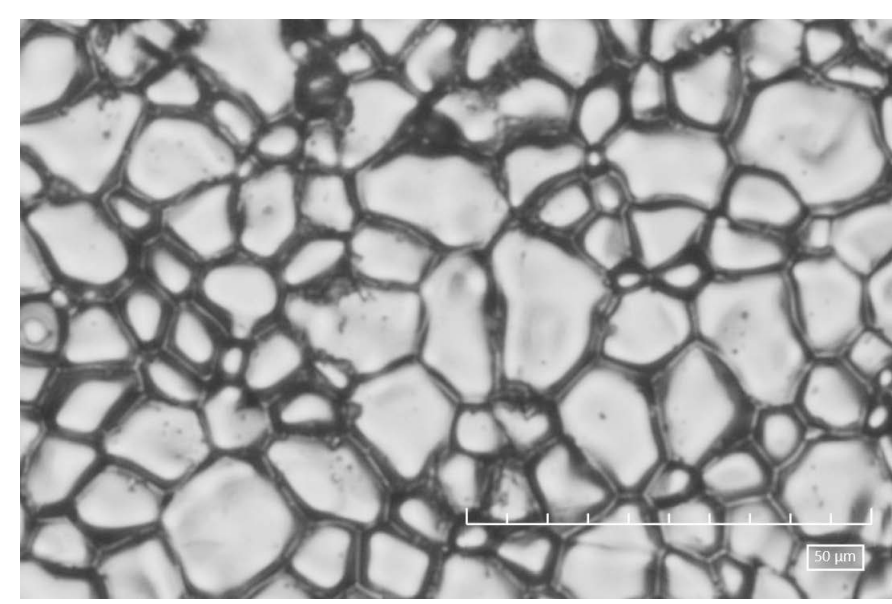

Fig. 2. Optical micrograph of the surface of an $\mathrm{Mn}-\mathrm{Zn}$ ferrite core. The scale has divisions of $5 \mu \mathrm{m}$. The darker regions between grains are due the roughness of the surface between grains and to the boundary phase.

pendent as described in [12] and [13].

To overcome this limitation, the influence of the material structure on the frequency scaling of the electro-magnetic parameters due to the sintered nature of ferrite cores is investigated in this paper. Due to the two-phases system caused by the sintering [1], a meta-material behaviour occurs, so that the overall characteristics become frequency dependent [12]. Based on this, a model of the overall conductivity and permittivity as function of frequency is introduced. Then, the phenomenon of standing waves in ferrite cores is investigated and its modelling for ring cores is summarized. With this, an eddy current loss model for ring cores is derived, based on the solution to wave equations in ring cores and using the electrical model of the material structure to calculate the permittivity and conductivity. Finally, the model is validated by measurements and the results are discussed.

\section{EQUiVALENT ELECTRICAL MODEL OF MATERIAL STRUCTURE}

The sintering process for ferrite cores results in an heterogeneous micro-structure. At high frequency, this heterogeneity has an impact on the electrical behaviour of the material.

\section{A. Ferrite material structure}

Ferrite cores are typically made by sintering [1] magnetic oxides (Iron, Zinc, Manganese or Nickel oxides) with conductivity lowering additives (Calcium oxide and/or Silicon dioxide) [14].

The properties of the final product depend not only on the mixture of the materials but also on the sintering conditions. The duration and the conditions of the different steps of the sintering have an impact on the final microstructure, both in terms of the size of the grains and of their shape but also of their inner composition [15]. Calcium and silicon oxides tend to migrate to the boundary of the grains, creating a thin layer with high resistivity while reducing the resistivity of the inner part of the grain. A high resistivity also implies that the relative impact of the permittivity is greater. a)

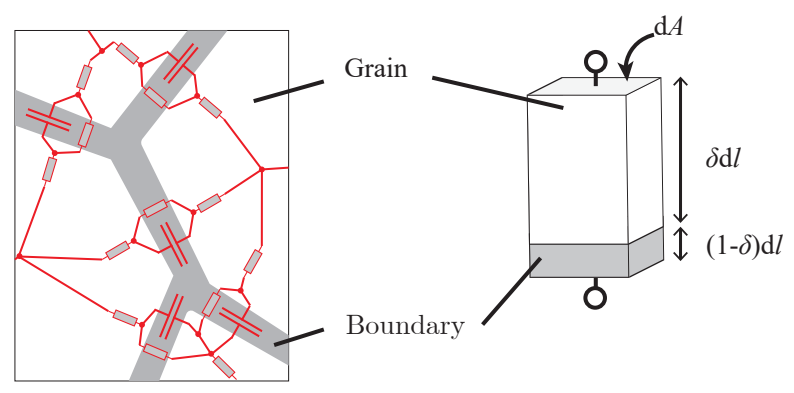

b)

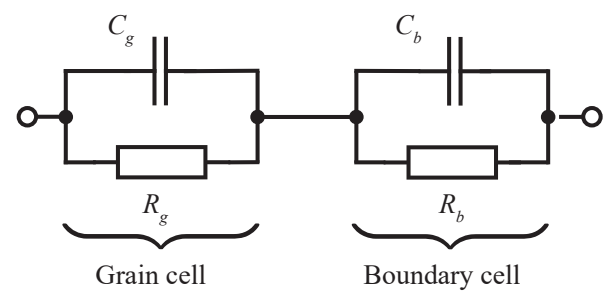

Fig. 3. Equivalent electrical model: a) conceptual description and 1D approximation of the structure, b) equivalent electrical circuit.

Overall, this results in two phases: The grains with high conductivity and the boundary layers with high resistivity and non-negligible permittivity. The macroscopic characteristics of the material are a combination of the parameters of the two phases. A model of those characteristics is derived in the following.

\section{B. Electrical model of the material structure}

The boundary layer acts as a capacitor surrounding a conductive grain (cf. Fig 3.a). Qualitatively, this means that at high frequencies, the impedance of the boundary layer decreases, resulting in an effective short-circuit of the insulating nature of the layer.

As proposed in [12], the two phases of the model can be represented using a 1D model (cf. Fig 3.a). Each phase of the material is described by an electrical cell made of the parallel connection of a resistor and a capacitor (cf. Fig 3.b). The two cells have the following impedances:

$$
\begin{aligned}
& \underline{Z}_{b}(\omega)=R_{b} / / \frac{1}{\mathrm{j} \omega C_{b}}=\frac{\mathrm{d} l(1-\delta)}{\mathrm{d} A\left(\sigma_{b}+\mathrm{j} \omega \epsilon_{b}\right)} \\
& \underline{Z}_{g}(\omega)=R_{g} / / \frac{1}{\mathrm{j} \omega C_{g}}=\frac{\mathrm{d} l \delta}{\mathrm{d} A\left(\sigma_{g}+\mathrm{j} \omega \epsilon_{g}\right)}
\end{aligned}
$$

Where $\sigma_{b}$ and $\sigma_{g}$ are the conductivities of the boundary layer and of the inner part of the grain. $\epsilon_{b}$ and $\epsilon_{g}$ are the permittivities of the boundary layer and of the inner part of the grain. $\delta$ is the proportion of the inner grain phase in the material (cf. Fig. 3.a). $\mathrm{d} A$ and $\mathrm{d} l$ are the cross section and the length of the infinitesimal element (cf. Fig. 3.a). Due to the series connection of the two cells, the total impedance is:

$$
\begin{aligned}
\underline{Z}_{t o t}(\omega) & =Z_{t o t, D C} \frac{1+\mathrm{j} \omega \frac{\delta\left(\epsilon_{b}-\epsilon_{g}\right)+\epsilon_{g}}{\delta\left(\sigma_{b}-\sigma_{g}\right)+\sigma_{g}}}{\left(1+\mathrm{j} \omega \frac{\epsilon_{b}}{\sigma_{b}}\right)\left(1+\mathrm{j} \omega \frac{\epsilon_{g}}{\sigma_{g}}\right)} \\
Z_{t o t, D C} & =\frac{\mathrm{d} l}{\mathrm{~d} A} \frac{\delta\left(\sigma_{b}-\sigma_{g}\right)+\sigma_{g}}{\sigma_{b} \sigma_{g}}
\end{aligned}
$$


Dividing the effective capacitance and conductance by the ratio of the cross section over the length gives the effective permittivity and conductivity of the material:

$$
\begin{aligned}
\sigma_{e f f}(\omega) & =\Re\left(\frac{1}{\underline{Z}_{t o t}}\right) \frac{\mathrm{d} l}{\mathrm{~d} A} \\
& =\sigma_{e f f, D C} \cdot \frac{1-(\mathrm{j} \omega)^{2} \frac{\delta\left(\epsilon_{b}^{2} \sigma_{g}-\sigma_{b} \epsilon_{g}^{2}\right)+\sigma_{b} \epsilon_{g}^{2}}{\delta\left(\sigma_{b}^{2} \sigma_{g}-\sigma_{b} \sigma_{g}^{2}\right)+\sigma_{b} \sigma_{g}^{2}}}{1-(\mathrm{j} \omega)^{2} \frac{\left(\delta\left(\epsilon_{b}-\epsilon_{g}\right)+\epsilon_{g}\right)^{2}}{\left(\delta\left(\sigma_{b}-\sigma_{g}\right)+\sigma_{g}\right)^{2}}} \\
\sigma_{e f f, D C} & =\frac{\delta\left(\sigma_{b}^{2} \sigma_{g}-\sigma_{b} \sigma_{g}^{2}\right)+\sigma_{b} \sigma_{g}^{2}}{\left(\delta\left(\sigma_{b}-\sigma_{g}\right)+\sigma_{g}\right)^{2}} \\
\epsilon_{e f f}(\omega) & =\frac{\Im\left(\frac{1}{\underline{Z}_{t o t}}\right)}{\omega} \frac{\mathrm{d} l}{\mathrm{~d} A} \\
& =\epsilon_{e f f, D C} \frac{1-(\mathrm{j} \omega)^{2} \frac{\delta\left(\epsilon_{b}^{2} \epsilon_{g}-\epsilon_{b} \epsilon_{g}^{2}\right)+\epsilon_{b} \epsilon_{g}^{2}}{\delta\left(\sigma_{b}^{2} \epsilon_{g}-\epsilon_{b} \sigma_{g}^{2}\right)+\epsilon_{b} \sigma_{g}^{2}}}{1-(\mathrm{j} \omega)^{2} \frac{\left(\delta\left(\epsilon_{b}-\epsilon_{g}\right)+\epsilon_{g}\right)^{2}}{\left(\delta\left(\sigma_{b}-\sigma_{g}\right)+\sigma_{g}\right)^{2}}} \\
\epsilon_{e f f, D C} & =\frac{\delta\left(\sigma_{b}^{2} \epsilon_{g}-\epsilon_{b} \sigma_{g}^{2}\right)+\epsilon_{b} \sigma_{g}^{2}}{\left(\delta\left(\sigma_{b}-\sigma_{g}\right)+\sigma_{g}\right)^{2}}
\end{aligned}
$$

Based on these equations, the equivalent electric parameters can be obtained for different frequencies.

\section{FLUX DISTRIBUTION AS FUNCTION OF FREQUENCY}

At high frequencies, the coupling between electric and magnetic fields is not negligible, so that standing waves can occur and that eddy currents can be induced.

\section{A. Standing waves in ferrite cores}

As discussed above (see also [11] and [16]), ferrites have non negligible permittivity and conductivity. Therefore, standing electromagnetic waves can appear in the core within frequency ranges of interest, modifying the distribution of the magnetic flux within the core. Due to the changing flux distribution, eddy currents (cf. Fig 4) can then be induced and the permeance of the core could vary.

In case of ring cores, the induced current flows in concentric shells around the center (cf Fig 4.d), if only the first mode of standing waves is excited. Based on that, [17] presents a lumped modelling approach based on a ladder network of capacitive and inductive elements, each level of the ladder representing a concentric shell.

However, such a modelling method assumes that no mode of order higher than one can be excited. Modelling those higher modes requires to solve the Maxwell equations. Some geometries, such as ring and pot cores, present axial symmetry, which reduces the dimension of the problem to two.

\section{B. Flux distribution and current density in ring cores}

The present work focuses on ring cores in order to give a comprehensive understanding of eddy current losses in ferrite cores but a similar reasoning could be applied to other core shapes.
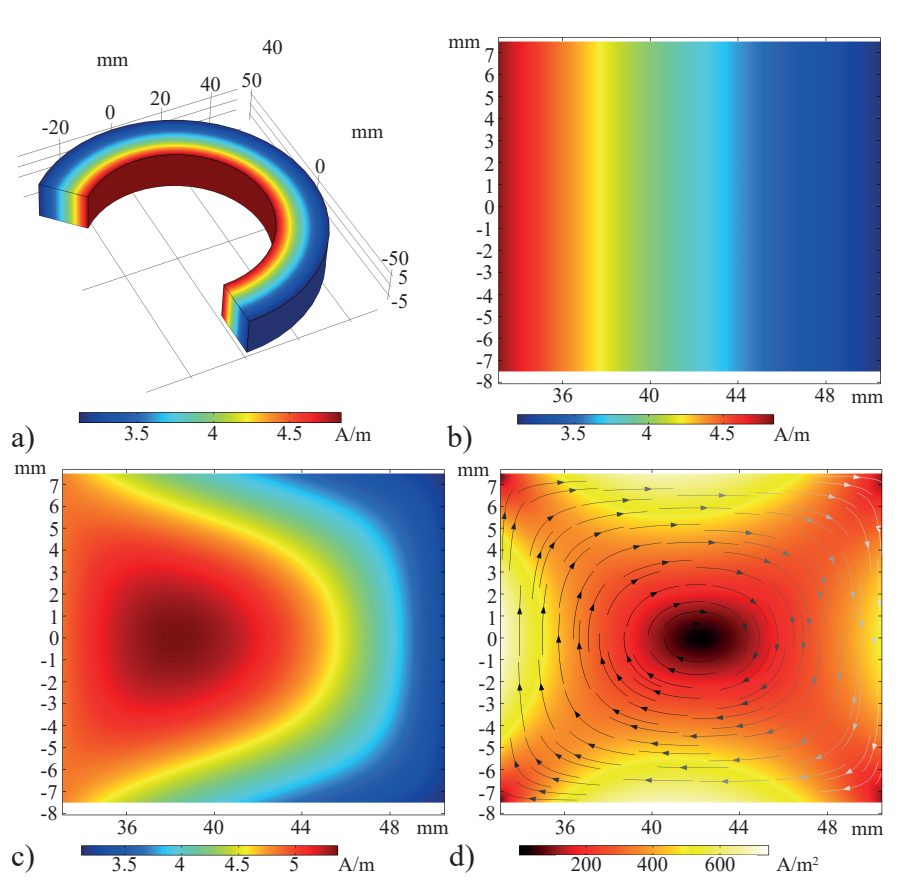

Fig. 4. FEM simulation of a core with a total current flowing around the core of $1 \mathrm{~A}$ (inner diameter: $65.8 \mathrm{~mm}$, outer diameter: $102 \mathrm{~mm}$, height: $15 \mathrm{~mm}$, relative permeability: 2900 , relative permittivity: 54000 , conductivity: $1.8 \mathrm{~S}$ ): a) $\mathrm{H}$-field at $5 \mathrm{~Hz}$. b) $\mathrm{H}$-field at $5 \mathrm{~Hz}$ (cross section). c) $\mathrm{H}$-field at $500 \mathrm{kHz}$ (cross section). d) Current density and current lines at $500 \mathrm{kHz}$ (cross section), mainly the first mode is excited and the current flows in concentric shells.

The complete wave equation for a ring core is derived in [10] and the main results are:

$$
\underline{\hat{H}}(\rho, z)=\frac{N_{w} \hat{\hat{i}}}{2 \pi}\left[\frac{\cosh (\gamma z)}{\cosh \left(\gamma \frac{h}{2}\right) \rho}+\sum_{n=1}^{\infty} \zeta_{n}\left(q_{1 n} \rho\right) \cos \left(p_{1 n} z\right)\right]
$$

with:

$$
\begin{aligned}
\zeta_{n} & =A_{n} \mathrm{I}_{1}\left(q_{1 n} \rho\right)+B_{n} \mathrm{~K}_{1}\left(q_{1 n} \rho\right) \\
A_{n} & =\frac{\frac{4}{h p_{1 n}}(-1)^{n+1} \frac{\gamma^{2}}{p_{1 n}^{2}+\gamma^{2}}\left(\frac{\mathrm{K}_{1}\left(q_{1 n} b\right)}{a}-\frac{\mathrm{K}_{1}\left(q_{1 n} a\right)}{b}\right)}{\mathrm{I}_{1}\left(q_{1 n} a\right) \mathrm{K}_{1}\left(q_{1 n} b\right)-\mathrm{I}_{1}\left(q_{1 n} b\right) \mathrm{K}_{1}\left(q_{1 n} a\right)} \\
B_{n} & =\frac{\frac{4}{h p_{1 n}}(-1)^{n+1} \frac{\gamma^{2}}{p_{1 n}^{2}+\gamma^{2}}\left(\frac{\mathrm{I}_{1}\left(q_{1 n} a\right)}{b}-\frac{\mathrm{I}_{1}\left(q_{1 n} b\right)}{a}\right)}{\mathrm{I}_{1}\left(q_{1 n} a\right) \mathrm{K}_{1}\left(q_{1 n} b\right)-\mathrm{I}_{1}\left(q_{1 n} b\right) \mathrm{K}_{1}\left(q_{1 n} a\right)} \\
p_{1 n} & =\frac{(2 n-1) \pi}{h} \\
q_{1 n}^{2} & =\gamma^{2}+p_{1 n}^{2} \\
\gamma^{2} & =\mathrm{j} \omega \mu_{D C}\left(\sigma_{e f f}(\omega)+\mathrm{j} \omega \epsilon_{e f f}(\omega)\right)
\end{aligned}
$$

Where $N_{w}, i, h, a, b, \sigma_{e f f}, \epsilon_{e f f}$ and $\mu_{D C}$ are the number of turns of the coil, the current in the coil, the height of the core, its inner diameter, outer diameter, effective conductivity, effective permittivity, and DC permeability, respectively. $\rho$ and $z$ are the radial and vertical coordinates. $\mathrm{I}_{1}$ and $\mathrm{K}_{1}$ denote the modified Bessel functions of order 1 of the first and second kind.

With (8), the flux density and the current density are obtained by:

$$
\begin{aligned}
& \underline{\hat{B}}(\rho, z)=\mu \underline{\hat{H}}(\rho, z) \\
& \underline{\hat{J}}(\rho, z)=\nabla \times \underline{\hat{H}}(\rho, z)
\end{aligned}
$$


Knowing the distribution of the magnetic field including both dielectric and resistive effects allows finally to derive the eddy current losses in the core.

\section{PROPOSED EDDY CURRENT LOSS MODEL}

By taking the permittivity and conductivity as calculated in Section II-B and using those values as inputs for the wave equation described in Section III-B, a new model is derived in the following. Multiplying (8) by the permeability and integrating the result over the cross section $A$ results in the magnetic flux:

$$
\begin{aligned}
& \hat{\phi}=\mu \iint_{A} \underline{\hat{H}}(\rho, z) \mathrm{d} A \\
& \hat{\phi}=\mu \frac{N_{w} \hat{\hat{i}}}{2 \pi}\left[\frac{2}{\gamma} \tanh \left(\frac{\gamma h}{2}\right) \ln \left(\frac{b}{a}\right)+\sum_{n=1}^{\infty} \Gamma_{n}\right]
\end{aligned}
$$

with:

$$
\begin{aligned}
\Gamma_{n} & =\frac{2\left(\xi_{n}+\chi_{n}\right)(-1)^{n-1}}{p_{1 n}} \\
\xi_{n} & =\frac{A_{n}}{q_{1 n}}\left(\mathrm{I}_{0}\left(q_{1 n} b\right)-\mathrm{I}_{0}\left(q_{1 n} a\right)\right) \\
\chi_{n} & =\frac{B_{n}}{q_{1 n}}\left(\mathrm{~K}_{0}\left(q_{1 n} b\right)-\mathrm{K}_{0}\left(q_{1 n} a\right)\right)
\end{aligned}
$$

$\mathrm{I}_{0}$ and $\mathrm{K}_{0}$ are the modified Bessel functions of order 0 of the first and second kind. One can then define an equivalent permeance:

$$
\underline{\lambda}=\frac{\hat{\underline{\phi}}}{N_{w} \underline{\hat{i}}}=\frac{\mu}{2 \pi}\left[\frac{2}{\gamma} \tanh \left(\frac{\gamma h}{2}\right) \ln \left(\frac{b}{a}\right)+\sum_{n=1}^{\infty} \Gamma_{n}\right]
$$

Eventually, eddy current losses are obtained by:

$$
P_{e c}=\Re\left(\frac{\hat{\hat{\mathrm{u}}}}{2}\right)=\Re\left(\frac{\mathrm{j} \omega \hat{\phi} \hat{\theta}^{*}}{2}\right)=\Re\left(\frac{\mathrm{j} \omega \hat{\phi}^{2}}{2 \underline{\lambda}^{*}}\right)
$$

with $\underline{\hat{u}}$ and $\underline{\hat{\theta}}$ being the excitation voltage and the magnetic potential. $\underline{\alpha}^{*}$ indicates the complex conjugate of $\underline{\alpha}$. In that way, the eddy current losses can be obtained analytically.

\section{EXPERIMENTAL VALIDATION}

In order to highlight the loss predicting capabilities of the model and discuss its behaviour, an experimental comparison is run.

\section{A. Measurement setup}

The model is validated using an N87 ferrite core with an outer diameter of $102 \mathrm{~mm}$. The loss and the volume averaged permeability are measured using a BH-analyzer IWATSU SY-8219 under sinusoidal excitation. Measurements are performed from 10 to $700 \mathrm{kHz}$ for different peak-to-peak effective flux densities $\Delta B$, whenever the loss density is under $1000 \mathrm{~kW} \mathrm{~m}^{-3}$. The loss density is kept under this threshold to avoid significant heating of the sample. All measurements are performed at room temperature.
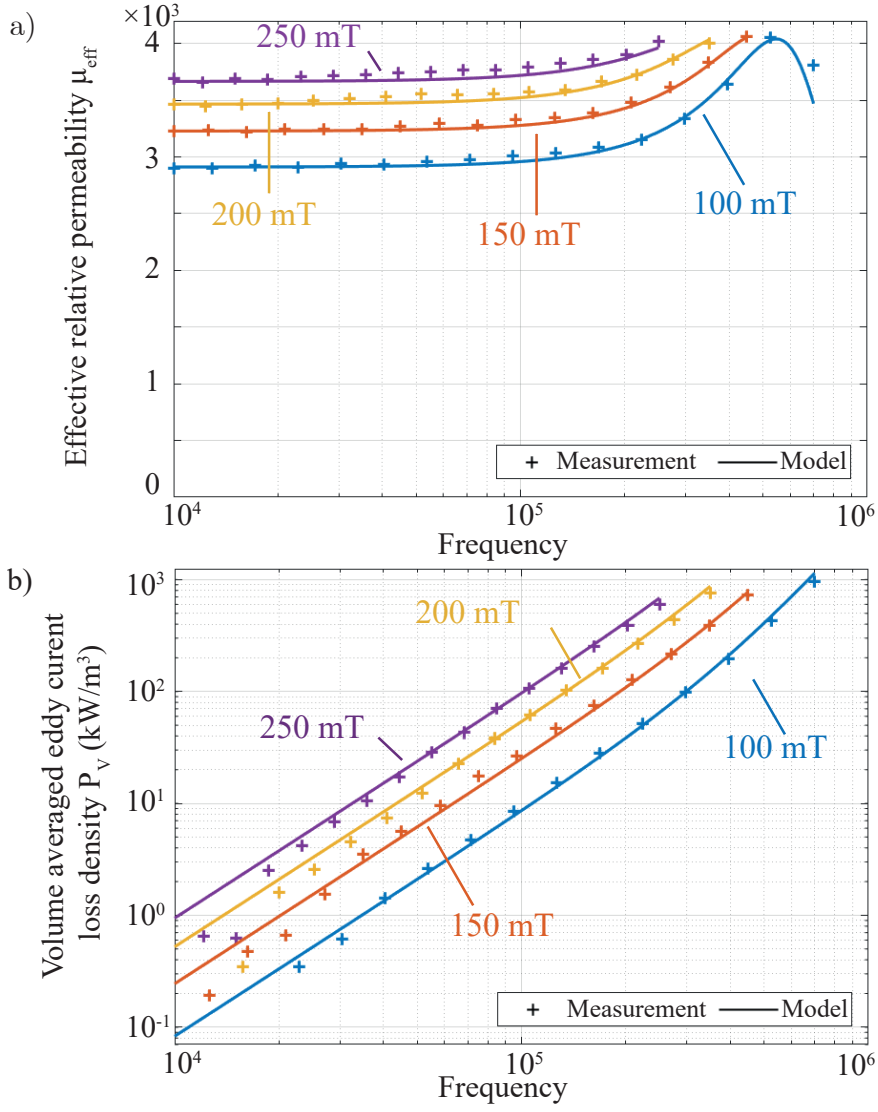

Fig. 5. Comparison of the model with the measurement results: a) effective relative permeability and b) volume averaged eddy current loss density. The values of the effective flux density are given as peak-to-peak.

\section{B. Model implementation}

The proposed model is implemented in MATLAB. The permeability is taken from low frequency measurements, for each value of excitation. The other parameters of the model (permittivities and conductivities of the grain and of the boundary as well as the proportion of inner grain phase in the material) are obtained by fitting the model to the measurements.

\section{Results}

The effective permeability $\underline{\mu}_{e f f}$ is derived as:

$$
\underline{\mu}_{e f f}=\underline{\lambda} \frac{l_{e}}{A_{e}}
$$

Where $A_{e}$ and $l_{e}$ are the effective cross section and magnetic path length, defined according to [18]. The eddy current losses calculated with the proposed model are compared with the difference between the measured losses and the hysteresis losses. The hysteresis losses are taken from low frequency measurements, considering the linear dependence of losses on frequency below $20 \mathrm{kHz}$ (cf. Fig 1) and then scaled linearly with frequency. The result is expressed in terms of volume averaged loss density as defined in [18]:

$$
\frac{P_{e c, e s t}(f)}{V_{e}}=\frac{P_{\text {loss }}(f)-P_{\text {loss }}\left(f_{0}\right) \frac{f}{f_{0}}}{V_{e}}
$$


TABLE I

FitTed MATERIAL PARAMETERS (N87)

\begin{tabular}{|c|c|c|c|c|c|}
\hline$\Delta B(\mathrm{mT})$ & $\delta$ & $\sigma_{g}(\mathrm{~S})$ & $\sigma_{b}(\mathrm{mS})$ & $\epsilon_{g} / \epsilon_{0}$ & $\epsilon_{b} / \epsilon_{0}$ \\
\hline 100 & 0.9721 & 5.5 & 27.1 & 1 & $2.59 \cdot 10^{3}$ \\
\hline 150 & 0.9721 & 5.4 & 37.7 & 1 & $2.65 \cdot 10^{3}$ \\
\hline 200 & 0.9721 & 5.2 & 48.4 & 1 & $3.20 \cdot 10^{3}$ \\
\hline 250 & 0.9721 & 5.1 & 59.4 & 1 & $3.41 \cdot 10^{3}$ \\
\hline
\end{tabular}

Where $P_{\text {loss }}, P_{\text {ec,est }}, f, f_{0}$, and $V_{e}$ are the total measured losses of the core, the estimated eddy current losses of the core, the frequency, the frequency at which the hysteresis losses were measured, and the effective magnetic volume of the core. The eddy current loss density and the permeability from the measurements and from the model are displayed in Fig. 5. The fitted parameters are given in table I.

\section{Discussion}

As shown in Fig. 5, the proposed model agrees well with the measurements, capturing efficiently the frequency dependence of the permeability over almost 2 decades and the eddy current losses at frequencies where they are significant (cf. Fig. 1 for comparison with total losses). Moreover, the shape of the permeability curve is similar to a capacitive resonance, confirming the modelling approach.

The dependence of the parameters on the flux density (cf. table I) could be caused by quantum tunneling, as proposed in [19] to explain the non-linearity of the boundary layer conductivity. This phenomenon implies that the conductivity is dependent on the applied electric field, which is a function of the product of flux density and frequency in magnetic cores. The obtained parameters match nevertheless well with values found in literature. The conductivity of the grain is in accordance with [20] and [21]. The overall permittivity is of similar magnitude as values found in [13]. Combining a typical grain size of 10 to $25 \mu \mathrm{m}$ (cf. Fig. 2) with the obtained proportion of the inner grain phase $\delta=0.972$, the boundary thickness is estimated from 0.3 to $0.7 \mu \mathrm{m}$. A chemical composition analysis of the boundary in [15] gives a thickness of $0.5 \mu \mathrm{m}$, close to the estimated value. However, a sub-nanometer thickness is also given in [21]. It should be noted that the material model used in the present article implies that a thick boundary layer with higher conductivity and permittivity could produce the same output as a thin layer with lower conductivity and permittivity. Removing this uncertainty requires more advanced modelling of the material.

This model also gives a physical interpretation to Bertotti's excess losses. The presence of wave mode excitation in the core implies that the frequency dependence of eddy current is more than quadratic. This dependence is further reinforced by the increase in apparent conductivity with frequency. Therefore, the complete Steinmetz equation cannot model eddy current losses associated with high frequencies, so that the additional term of Bertotti model is necessary.

\section{CONCLUSION}

A new core loss model based on a meta-material modelling approach of the material parameters and on the wave equations in the core is presented for estimating the eddy current core losses in ferrite ring cores under sinusoidal excitation. Combined with a state-of-the-art estimation of hysteresis losses, this model enables an accurate estimation of the total core losses in ring cores under sinusoidal excitation over a wide range of frequency (10 to $700 \mathrm{kHz}$ ). Additionally, it provides accurate prediction of the effective permeability against frequency. This approach explains also the over-quadratic dependence of eddy current losses in ferrite cores.

\section{REFERENCES}

[1] A. Goldman, Modern Ferrite Technology. Springer US, 2006.

[2] C. A. Quinn, "Empowering the electronics industry: A power technology roadmap," CPSS Transactions on Power Electronics and Applications, vol. 2, no. 4, pp. 306-319, Dec. 2017.

[3] M. Stojadinović and J. Biela, "Modeling and design of a mediumfrequency transformer for high-power DC-DC converters," IEEJ Journal of Industry Applications, vol. 8, no. 4, pp. 685-693, jul 2019.

[4] C. P. Steinmetz, "On the law of hysteresis (part II.) and other phenomena of the magnetic circuit," Transactions of the American Institute of Electrical Engineers, vol. IX, no. 1, pp. 619-758, Jan. 1892.

[5] J. Mühlethaler, "Modeling and multi-objective optimization of inductive power components," Ph.D. dissertation, ETHZ, 2012.

[6] G. Bertotti, "General properties of power losses in soft ferromagnetic materials," IEEE Transactions on Magnetics, vol. 24, no. 1, pp. 621-630, Jan. 1988.

[7] M. Mu, "High frequency magnetic core loss study," Ph.D. dissertation, Virginia Tech, 2013.

[8] I. Mayergoyz, Mathematical Models of Hysteresis and Their Applications. Elsevier, 2003.

[9] D. Jiles, "Modelling the effects of eddy current losses on frequency dependent hysteresis in electrically conducting media," IEEE Transactions on Magnetics, vol. 30, no. 6, pp. 4326-4328, 1994.

[10] W. Hauser, "Modellbildung für strukturabhängige Effekte in Ferritkernen," Ph.D. dissertation, Friedrich-Alexander-Universität ErlangenNürnberg (FAU), 2018.

[11] M. Kacki, M. S. Rylko, J. G. Hayes, and C. R. Sullivan, "A study of flux distribution and impedance in solid and laminar ferrite cores," in Applied Power Electronics Conference and Exposition (APEC), Mar. 2019.

[12] C. G. Koops, "On the dispersion of resistivity and dielectric constant of some semiconductors at audiofrequencies," Physical Review, vol. 83, no. 1, pp. 121-124, Jul. 1951.

[13] M. Kacki, M. S. Rylko, J. G. Hayes, and C. R. Sullivan, "A practical method to define high frequency electrical properties of MnZn ferrites," in Applied Power Electronics Conference and Exposition (APEC), Mar. 2020.

[14] C. Guillaud, M. Paulus, and R. Vautier, "La diminution des pertes par courant de Foucault dans les ferrites de manganèse-zinc par addition de calcium," Comptes rendus hebdomadaires des Séances de l'Académie des Sciences, pp. 2712-2715, Jun. 1956.

[15] K. Ishino and Y. Narumiya, "Development of magnetic ferrites: Control and application of losses," Ceramic Bulletin, vol. 66, no. 10, pp. 1469 1474, 1987.

[16] G. Skutt, "High-frequency dimensional effects in ferrite-core magnetic devices," Ph.D. dissertation, Virginia Polytechnic Institute and State University, Oct. 1996.

[17] C. R. Sullivan, "Lumped model to explain and approximate dimensional effects in ferrite cores," in PSMA Magnetics Workshop, 2019. [Online]. Available: https://www.psma.com/2019-magnetics-workshoppresentations

[18] Calculation of the effective parameters of magnetic piece parts, IEC 60205:2016, International Electrotechnical Commission (IEC) Std., Nov. 2016.

[19] W. A. Roshen, "High-frequency tunneling magnetic loss in soft ferrites," IEEE Transactions on Magnetics, vol. 43, no. 3, pp. 968-973, Mar. 2007.

[20] Y. Fukuda, S. Nagata, and K. Echizenya, "Electrical conductivity of MnZn ferrite single crystals with a small number of $\mathrm{Fe} 2+$ ions," Journal of Magnetism and Magnetic Materials, vol. 279, no. 2-3, pp. 325-330, Aug. 2004.

[21] F. Fiorillo, C. Beatrice, O. Bottauscio, and E. Carmi, "Eddy-Current Losses in Mn-Zn Ferrites," IEEE Transactions on Magnetics, vol. 50, no. 1, Jan. 2014. 